\title{
Postirradiation pulmonary fibrosis complicated by aspergilloma and bronchocentric granulomatosis
}

\author{
H MAKKER, K MCCONNOCHIE, A R GIBBS \\ From the Department of Tuberculosis and Chest Diseases and Department of Pathology, University of Wales \\ College of Medicine, Llandough Hospital, Penarth, South Glamorgan
}

ABSTRACT An asthmatic patient requiring corticosteroid treatment developed a pulmonary aspergilloma in an area of postmastectomy radiation fibrosis. At necropsy bronchocentric granulomatosis was also found.

The major clinical syndromes associated with Aspergillus fumigatus are allergic (asthma, allergic bronchopulmonary aspergillosis, and extrinsic allergic alveolitis), saprophytic (mycetoma), and invasive. It is uncommon for these different forms to occur in the same patient.

\section{Case report}

A 52 year old woman, a non-smoker, with atopic asthma since childhood and mild rheumatoid arthritis, had an excision biopsy of the right breast in June 1986, which revealed adenocarcinoma. She received a course of radiotherapy. In December 1986 she complained of pleurisy and her chest radiograph showed a right upper zone opacity. The results of investigations, which included bronchoscopy, were normal except for a raised serum IgE concentration and aspergillus precipitins. She responded to prednisolone and was well until October 1987, when she again presented with pleurisy associated with cough and purulent sputum. On this occasion $A$ fumigatus was isolated from her sputum, precipitins were present, and serum IgE exceeded $1000 \mathrm{ng} / \mathrm{l}$. Her chest radiograph now showed an ill defined right upper zone opacity with contraction and distortion. Two weeks later there was evidence of a cavity and tomography suggested the presence of a mycetoma. Her condition deteriorated despite prednisolone and she required frequent courses of antibiotics. In January 1988 she developed an empyema, from which she died.

At necropsy there was bilateral pleural thickening with dense adhesions. The right upper lobe of the lung was almost completely destroyed by a large irregular cavity, containing brown friable material, which communicated with the pleural cavity. The middle and lower lobes were solid apart from three small cavities in the apical segment. The left lung showed multiple foci of bronchopneumonia but no other macroscopic abnormality. Microscopic examination of the

Address for reprint requests: Dr $\mathrm{K}$ McConnochie, Department of Tuberculosis and Chest Disease, Llandough Hospital, Penarth, South Glamorgan CF6 1XX.

Accepted 9 May 1989 lungs showed an aspergilloma in the right upper lobe, $\stackrel{\vec{A}}{A}$ extensive diffuse alveolar damage, and interstitial fibrosis in $\infty$ the middle and lower lobes, interpreted as radiation pneumonitis.

In the middle and lower lobes several small bronchi and 음 bronchioles showed eosinophilic necrosis with granulo- matous changes in their walls - that is, the changes of $D$ bronchocentric granulomatosis (figure); direct staining disclosed small numbers of aspergillus hyphae. The left lung $\frac{\bar{c}}{\omega}$ showed bronchopneumonia and small focal areas of interstitial fibrosis; the bronchi showed basement membrane thickening and muscular hypertrophy but no other asthmatic features.

\section{Discussion}

This patient's asthma, which began in childhood, may have $\stackrel{2}{\mathbb{D}}$ been a manifestation of allergic bronchopulmonary aspergillosis, though none of the features of this disorder was documented before her radiotherapy. Aspergilloma has been reported in postirradiation pulmonary fibrosis ${ }^{12}$ and possibly both irradiation and allergic bronchopulmonary aspergillosis predisposed to its development in this case. Bronchocentric granulomatosis may be a manifestation of 0 allergic bronchopulmonary aspergillosis, ${ }^{3}$ presumably owing $\stackrel{x}{x}$ to an immunological reaction to the aspergillus, but may $\bar{\sigma}$ occur in non-atopic individuals, when the pathogenesis is unknown. In this case it is likely to have been a consequence $\hat{\rho}$ of allergic bronchopulmonary aspergillosis as aspergillus hyphae were found in the lesions. Bronchocentric granulomatosis is uncommon and to the best of our knowledge has not been described in association with $D$ aspergilloma, though aspergilloma may follow allergic bronchopulmonary aspergillosis ${ }^{4-7}$ and vice versa. ${ }^{8}$

The changes of bronchocentric granulomatosis were? present in the middle and lower lobes of the right lung despite $N$ corticosteroid treatment, whereas active allergic bronchopul- N monary aspergillosis was not present in the left lung. The $\frac{\omega}{\sigma}$ change on the right might have resulted from continual leakage of aspergillus from the right upper lobe into the middle and lower lobe bronchi.

In the absence of massive haemoptysis medical man-? agement of aspergilloma is usually appropriate. In this case $\square$ there was no response to corticosteroid treatment, which may

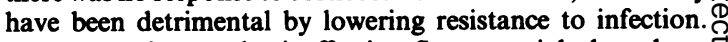
Ketoconazole was also ineffective. Surgery might have been $\stackrel{\Phi}{\Phi}$ useful, if only to remove the source of antigenic stimulus, $\frac{a}{\sigma}$ and-given the fatal outcome-we would recommend that in 


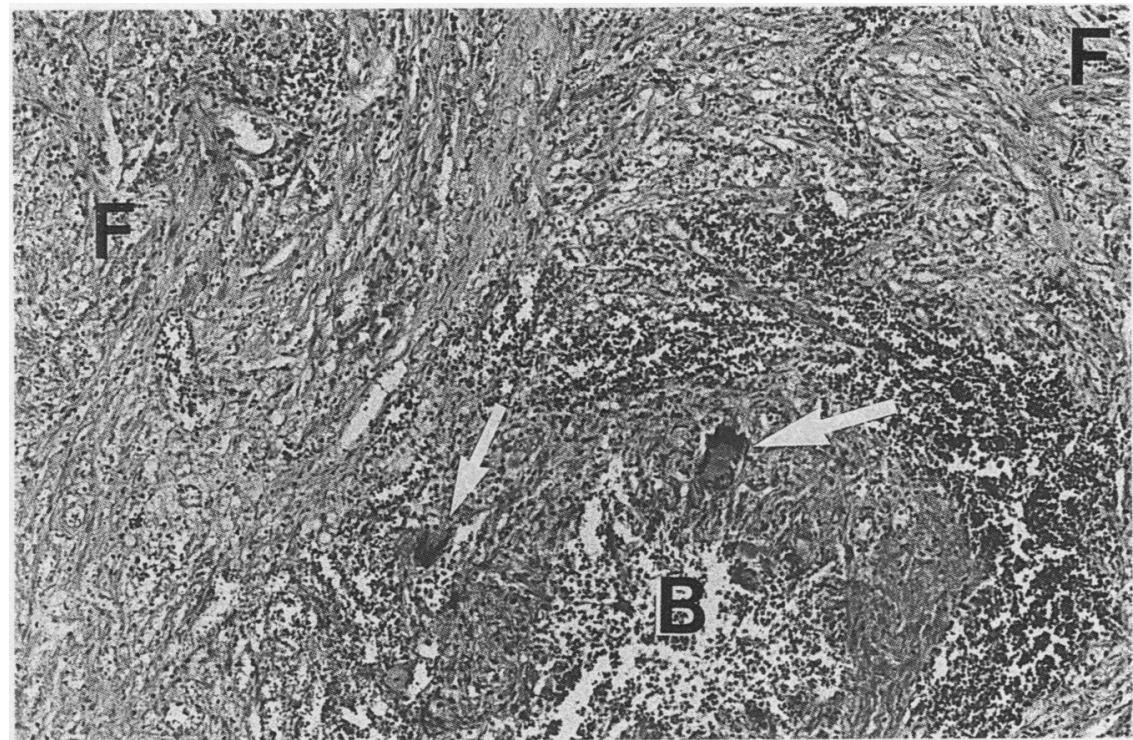

Lung tissue showing substantial fibrosis $(F)$ with an area of granulomatous inflammation containing giant cells (arrowed) centered on a bronchiole (B).

these circumstances consideration should be given to this approach.

\section{References}

1 Ward MJ, Davies D. Pulmonary aspergilloma after radiation therapy. Br J Dis Chest 1982;76:361-4.

2 Long JP, McErlean DP, FitzGerald MX. Changing patterns of pulmonary aspergilloma [abstract]. Thorax 1981;36:224.

3 Bosken CH, Myers JL, Greenberger PA, Katzenstein AA. Pathological features of allergic bronchopulmonary aspergillosis. Am $J$ Surg Pathol 1988;12:216-22.
4 Henderson AH. Allergic aspergillosis: review of 32 cases. Thorax 1968;23:501-12.

5 Safirstein BH. Aspergilloma consequent to allergic bronchopulmonary aspergillosis. Am Rev Respir Dis 1973;108:940-3.

6 Safirstein BH, D'Souza MF, Simon G, Tai EH-C, Pepys J. Five year follow-up of allergic bronchopulmonary aspergillosis. Am Rev Respir Dis 1973;108:450-9.

7 Davies D. Lung fibrosis and cavitation mimicking tuberculosis. Tubercle 1970;51:246-54.

8 Ein ME, Wallace RJ Jr, Williams TW Jr. Allergic bronchopulmonary aspergillosis-like syndrome consequent to aspergilloma. Am Rev Respir Dis 1979;119:811-20. 